\title{
Single Carrier Multi-Tone Modulation Scheme
}

\author{
Roman M. Vitenberg \\ Guarneri Communications Ltd, Israel \\ roman@guarneri-communications.com
}

\begin{abstract}
-
In this paper, we propose a modulation scheme, which can improve the performance of a variety of wired and wireless communication systems.

We call this scheme "Single Carrier Multi-Tone" (SCMT) because it is a name well describes the physical principles on which it is made. This scheme was developed based on a study known SC-FDMA, OFDM and SC-FDE technologies. The aim of this research was to improve the performance of existing communication systems for terrestrial television broadcasting. The proposed modulation scheme combines the advantages of known technologies and devoid of their shortcomings. The several key characteristics of the SCMT are illustrated by the results of MATLAB simulation.
\end{abstract}

Keywords - SC FDMA, OFDM, TV Broadcasting, SC FDE, ATSC, PAR, QAM, VSB, multipath, single carrier, Multi-tone.

\section{INTRODUCTION}

Orthogonal Frequency Division Multiplexing (OFDM) is the most popular transmission technology in digital terrestrial broadcasting (DTTB), adopted by many DTTB standards.

The big advantage of OFDMA is its robustness in the presence of multipath signal propagation [2]. The immunity to multipath arises from the fact that the OFDMA system transmits information to the $M$ orthogonal frequency carriers, each of which operates at $1 / M$ times the bit rate of the information signal. On the other hand, the OFDMA waveform exhibits noticeable envelope fluctuations resulting in a high peak-to-average power ratio (PAPR). Signals with a high PAPR require highly linear power amplifiers to avoid excessive intermodulation distortion. To achieve this linearity, the amplifiers have to operate with a large back off from their peak power. The result is low power efficiency (measured by-the ratio of transmitting power to the power dissipated), which poses significant difficulties on portable wireless terminals.

Another problem with the OFDMA scheme in wireless transmissions derives from the inevitable offset in frequency references among the transmitted and receiver terminals. Frequency offset destroys the orthogonality of the transmissions, thus introducing subcarriers interference.

To overcome these drawbacks, 3GPP is studying a modified form of OFDMA for uplink transmissions in the "long-term evolution (LTE)" of cellular systems [12] - [15]. A modified version of OFDMA, called Single Carrier FDMA (SC-FDMA), has been described in several standard documents $[13,14]$ and researches $[1,12]$. As in OFDMA, the transmitter in the SC-FDMA uses number orthogonal frequencies (subcarriers) to transmit information symbols. Compared to OFDMA, the mechanism significantly reduces variations in the envelope of the transmitted signal. Therefore, SC-FDMA signals have inherent lower PAPR then OFDMA signals [1].

Nevertheless, in cellular systems with severe multipath, SC-FDMA signals are sent to the base station with substantial inter-symbol interference. The base station uses an adaptive equalization in the frequency domain to cancel the interference. This arrangement makes sense in a cellular system because it reduces the distortions of the linear amplification in the portable terminal, at the cost of complexity of signal processing (frequency domain equalization) on the base station.

A similar occurs in the design terrestrial TV broadcasting systems. The great importance in such systems has the efficiency of RF transmitters.

Currently for terrestrial TV broadcasting systems is being used a single carrier 8VSB system in the United States (ATSC A/53) and OFDM system in Europe (DVB-T, DVB-T2) and Asia (ISDB-T, DTMB-T).

The OFDM DVB standard provides a large number of configurations, and useful features but requires a transmitter peak power at $6 \mathrm{~dB}$ more than the peak power of the ATSC transmitter $\mathrm{A} / 53$. As a result, the benefits of DVB, obtained through the use of the LDPC code, high constellation QAM256 and decrease the number of pilots, reduced.

The indisputable advantage of DVB is the high performance of communications in a multipath channel. Therefore, the DVB terrestrial TV broadcasting system provides better connectivity with mobile objects than A/53.

The logical solution would be to use a new SC FDMA modulation for future terrestrial TV Broadcasting systems, but it is possible only after a significant improvement in the SC FDMA technology. This paper is structured as follows: in the next section describes the SC FDMA modulation scheme and discusses its shortcomings, further in the third section will be described offered by us SCMT modulation scheme. The fourth section presents the results of MATLAB simulations. In conclusion, we describe the main results of this paper. 


\section{SC FDMA MODULATION SCHEME}

SC-FDMA transmitter converts the binary input signal into a sequence of modulated subcarriers. To do this, it performs the signal processing illustrated in Figure 1. Signal processing is performed during the repetitive time intervals called blocks. A block is the time used for the formation one SC FDMA symbol. During this interval, a system generates all subcarriers once. Several SC FDMA symbols combined in a transmission frame for continuous transmission.

The input binary sequence comes to the QAM Modulator, which converts binary entering into a multi-level sequence of complex numbers. The QAM modulator uses one of several possible modulation formats, including binary phase shift keying (BPSK), quaternary PSK (QPSK), 16 level quadrature amplitude modulation (16-QAM) and 64-QAM.

The system adapts the modulation format and thus the bit rate according to the current channel condition of the terminal.

The transmitter sends groups of the complex numbers $\mathrm{x}(\mathrm{t})$ by packets, each of which contains $\mathrm{N}$ symbols. In the first stage of the SC-FDMA modulation scheme is performed $\mathrm{N}$-points discrete Fourier transform (DFT), to get a frequency domain representation of the input symbols $X(f)$. Then, it maps each of N DFT outputs to one of inputs of M-point IDFT core $(\mathrm{M}>\mathrm{N})$. Others inputs of IDTF core are connected to zero. The IDFT core generates $\mathrm{M}$ orthogonal subcarriers, which may be transmitted over the channel. As in OFDMA, a typical value of $\mathrm{M}$ is 256 subcarriers and $\mathrm{N}=\mathrm{M} / \mathrm{Q}$ is an integer divisor of $\mathrm{M}$ (a typical value of $\mathrm{Q}$ is 16 ). $\mathrm{Q}$ is bandwidth expansion factor of the sequence of characters.

As in OFDMA, the M-point inverse DFT (IDFT) converts the amplitudes of the subcarriers into the time domain signal (SC FDMA symbol) Y (t). These symbols are transmitted sequentially.

The transmitter performs two other signal processing operations prior to transmission. It inserts a set of symbols referred to as a cyclic prefix $(\mathrm{CP})$ in order to provide a guard time to prevent inter-block interference (IBI) due to multipath propagation. .

If the length of the $\mathrm{CP}$ is longer than the maximum delay spread of the channel, or roughly, the length of the channel impulse response, then, there is no IBI. In general, CP is a copy of the last part of the block which is added at the beginning of each block for several reasons. . First, CP acts as a guard time between successive blocks. . Second, since CP is a copy of the last part of the block, it converts a discrete time linear convolution into a discrete time circular convolution.

Thus transmitted data propagating through the channel can be modeled as a circular convolution between the channel impulse response and the transmitted data block, which in the frequency domain is a point wise multiplication of the DFT frequency samples. Then, to remove the channel distortion, the DFT of the received signal can simply be divided by the DFT of the channel impulse response. A point-wise or a more sophisticated frequency domain equalization technique can be implemented to remove multi-path distortions.
The transmitter also performs a linear filtering operation referred to as pulse shaping in order to reduce out-of-band signal energy.

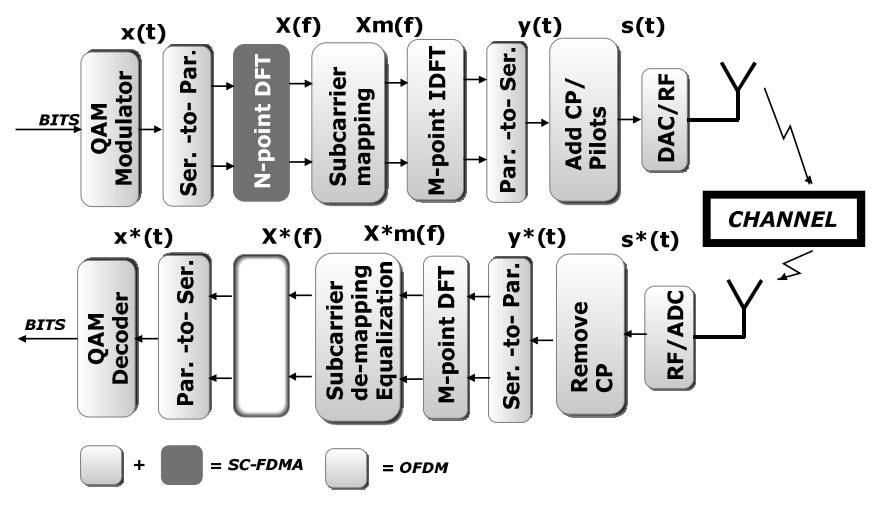

Figure 1: SC FDMA functional diagram.

OFDM has much in common with SC-FDMA. The only difference between SC FDMA and OFDM is the presence of the N-point DFT in the SC-FDMA transmitter and the $\mathrm{N}$-point IDFT in the SC-FDMA receiver. For this reason, SC-FDMA is sometimes referred to as DFT-spread OFDMA. Several approaches to mapping transmission symbols $X$ (f) to SC-FDMA subcarriers are currently under consideration. They are divided into two categories; distributed and localized. For terrestrial TV broadcasting, only localized transmission can be used.

In the localized subcarrier mapping mode, $\mathrm{N}$ consecutive subcarriers of IDFT are occupied by the DFT outputs of the input data resulting in a continuous spectrum that occupies a fraction of the total available bandwidth.

As it is well known, the main drawback of OFDM is the high level of peak-to-average power ratio (PARP). While PARP for the signal of a single carrier modulation is about $5 \sim 7 \mathrm{~dB}$, the PARP for OFDM signal is significantly higher (up to 12 $\mathrm{dB})$.

PARP in SC-FDMA system is significantly lower than in the OFDM because the transmitted signals have different statistical characteristics.

Suppose that we exclude cyclic prefixes from the transmitted signal and connect the outputs of DFT appropriate to firsts $\mathrm{N}$-inputs IDFT. In this case, the transmitted signal is equal to Q-times up sampled sequence of input QAM symbols. Evident that the transmitted signal will have the same statistical characteristics, including PARP, as the QAM input signal. As cyclic prefixes are represented segments of the transmitted signal $y(t)$ that their statistical characteristics the same as the signal $y(t)$. Therefore, PAPR of the transmitted signal $s(t)$ is the same as PAPR of the sequence of QAM symbols $x(t)$ passed through the output filter.

In the case of SC-FDMA modulation, the function of output filter is performed by FFT core. This filter has a rectangular shape and the bandwidth is exactly equal to $\mathrm{W}=\mathrm{N}^{*} \Delta \mathrm{F}$, where $\Delta \mathrm{F}$ is a frequency shift between subcarriers. 
This form of the spectrum is not optimal from the point of view of obtaining the minimal PAPR, but cannot be changed in the SC-FDMA scheme.

As a result the PAPR SC-FDMA output signal (in localized subcarrier mapping mode) is significant higher than in case of using optimal output filter [5].

Unlike OFDM SC-FDMA requires an additional $\mathrm{N}$-point DFT core in transmitter and $\mathrm{N}$-point IDFT core in the receiver.

Below we offer an improved system that overcomes the drawbacks of the SC-FDMA.

\section{Single Carrier Multi-Tone Modulation}

A functional diagram of the proposed communication system is shown in Figure 2. This modulation scheme which we called the SCMT is different from the well-known scheme SC-FDMA presence up-sample unit in the transmitter and down-sample unit in the receiver. Unlike SC FDMA Transmitter, which comprises N-point DFT core and M-point IDFT core, the SCMT Transmitter comprises M-point FFT and M-point IFFT cores. Respectively, the SC FDMA Receiver comprised M-point DFT core and N-point IDFT core, unlike the SCMT Receiver, which comprises M-point FFT core and M-point IFFT core.

. Since FFT and IFT transactions can be executed with the same FFT/IFFT core at different time intervals, the complexity of SCMT equipment is the same as for OFDM. Additional in SCMT transmitter included a programmable digital filter that allows us to change the shape of the spectrum of the transmitted signal with the purpose to obtain the minimum PAPR.

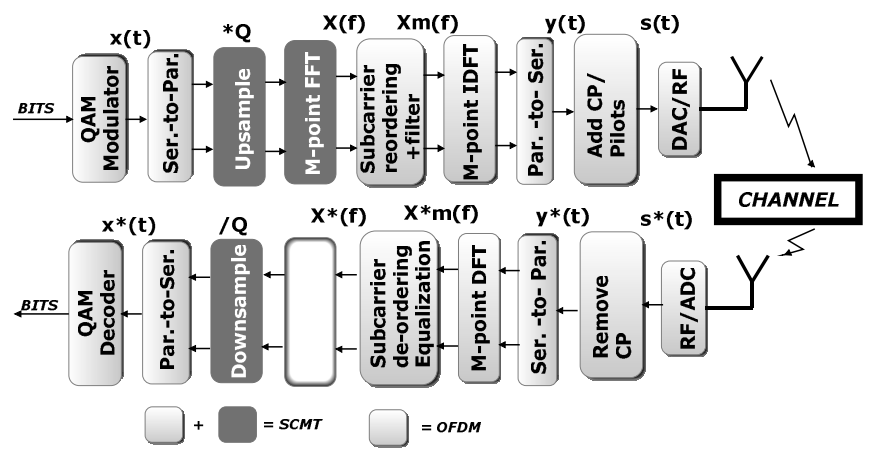

Figure 2: SCMT functional diagram.

In the proposed scheme as well as in SC-FDMA symbols generated by QAM modulator are combined into groups of $\mathrm{N}$ characters. These groups are converted into groups of $\mathrm{M}$ symbols by up-sampling. Up-sampling coefficient $Q=M / N$ is typically 2 or 4 depends from characteristics of RF filters in transmitter equipment. M-group characters on FFT output represent a mapping of up-sampled input signal into the frequency domain. In the frequency domain the up-sampled signal occupies bandwidth equal $\mathrm{Q}^{*} \mathrm{~W}$ and comprises $\mathrm{Q}$ copies of the input spectrum. Each FFT output is multiplied on coefficient that corresponds to a frequency characteristic of the optimal output filter. Filtered subcarriers are divided in two groups first of them occupied the lower part of spectrum, second occupied higher part of the spectrum. The reordering process performs relocation of these groups around selected carrier frequency. Wherein first group of subcarriers is placed above carrier frequency and the second group of subcarriers is placed below the carrier. Figure 3 shows the signal spectra at various stages of processing.

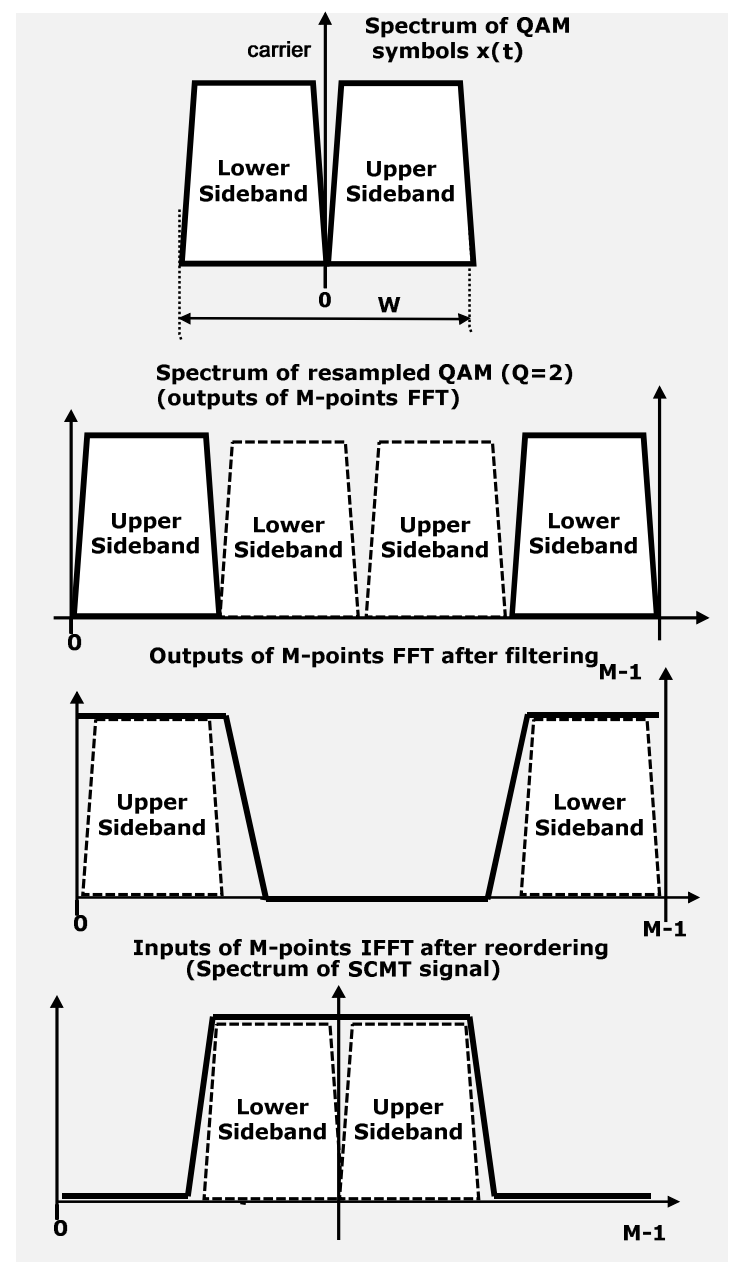

Figure 3: SCMT spectrum diagram.

The SCMT receiver performs an inverse allocation of subcarriers so the subcarriers above carrier frequency are shifted to the lower part of the spectrum and subcarriers below carrier frequency are placed at the end of the spectrum. After equalizing and re-ordering received subcarrier are processed by M-point IFFT. From the output of IFFT the groups of M-character comes to down-sampling unit. The 
down-sampling unit transforms these groups in $\mathrm{N}$-character packets of QAM symbols.

The proposed modulation scheme allows extensive opportunity to control the shape of the spectrum of the transmitted signal.

In particular, it is possible to create a single sideband communication system, the signal spectrum of which will correspond to the signal 8VSB. Such system can be fully backward compatible with a valid TV broadcast ATSC system. At the same time, thanks to using multi-tone technology this system can provide reliable communications with mobile devices. SCMT as OFDM are using precision equalization in the frequency domain, so both systems can use QAM modulation of high-level: 1024QAM and 4096QAM. In the case of VSB modulation, the SMNT system can operate with 16VSB, 32VSB and 64VSB signals. Therefore, the use of a single sideband SCMT modulating in future ATSC 3.0 standard TV Broadcast systems will significantly increase the speed of transmission of information.

Figure 4 shows a functional schematic of the SCMT system with single sideband transmission.

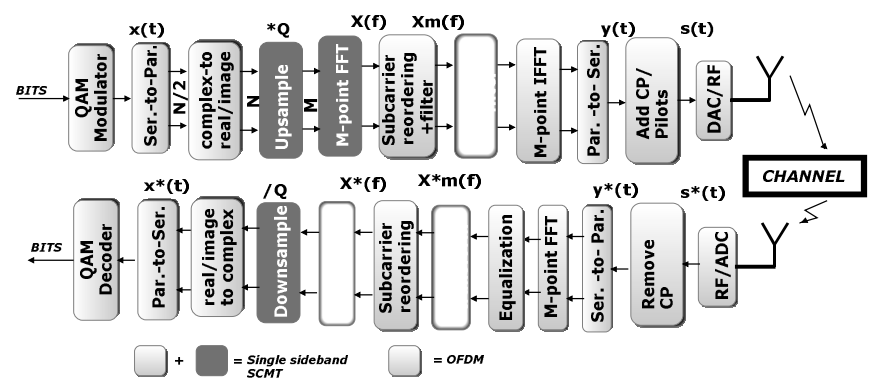

Figure 4: Single sideband SCMT functional diagram.

A single sideband SCMT transmitter comprises a sideband filter, which produces an output signal with the spectrum, which corresponds to the desired shape. In the case of an ATSC compatible broadcasting system, the sideband filter forms a spectrum of the output signal similar to a spectrum of $8 \mathrm{VSB}$ signal. Since the single side signal has a bandwidth $\mathrm{W} / 2$, then such a system transmits information with bitrate equal $1 / 2$ of the bitrate double side system. Therefore, the QAM symbols are packed in N/2-character group, each of them subsequently converted into a group of N-real numbers (PAM symbols).

The single sideband receiver includes Hilbert Transform unit, which restores the suppressed sideband. This unit is placed after an equalizer and before IFFT unit. As a result, on the output of IFFT are generated $M$ real numbers. After down-sampling $\mathrm{N}$ real numbers are converted into $\mathrm{N} / 2$ complex QAM symbols, which subsequently come to the input of QAM decoder. Figure 5 shows the signal spectrum in different processing stages.

As is clear from the functional diagram Figure 4 considered the system can operate in two modes, one with a single sideband modulation, for example VSB, and signal bandwidth W/2 and other with dual sideband QAM modulation and signal bandwidth $\mathrm{W}$.

This fact may be interesting for developers of a new ATSC 3.0 standard because allows to create a TV broadcast system that will be in one mode full compatible with existing $\mathrm{A} / 53$ and $\mathrm{A} / 153$ standards and 6-MHz television channel and in other mode will be used wide 12-MHz channel. Because of using multi-tone signals and high level of QAM constellations,

This system will provide aggregate bit rate up to 72 Mbps for 6-MHz channel and up to $144 \mathrm{Mbps}$ for the $12-\mathrm{MHz}$ channel.

The information bit rates will be corresponding up to 60 Mbps for 6-MHz channel and up to $120 \mathrm{Mbps}$ for the $12-\mathrm{MHz}$ channel.

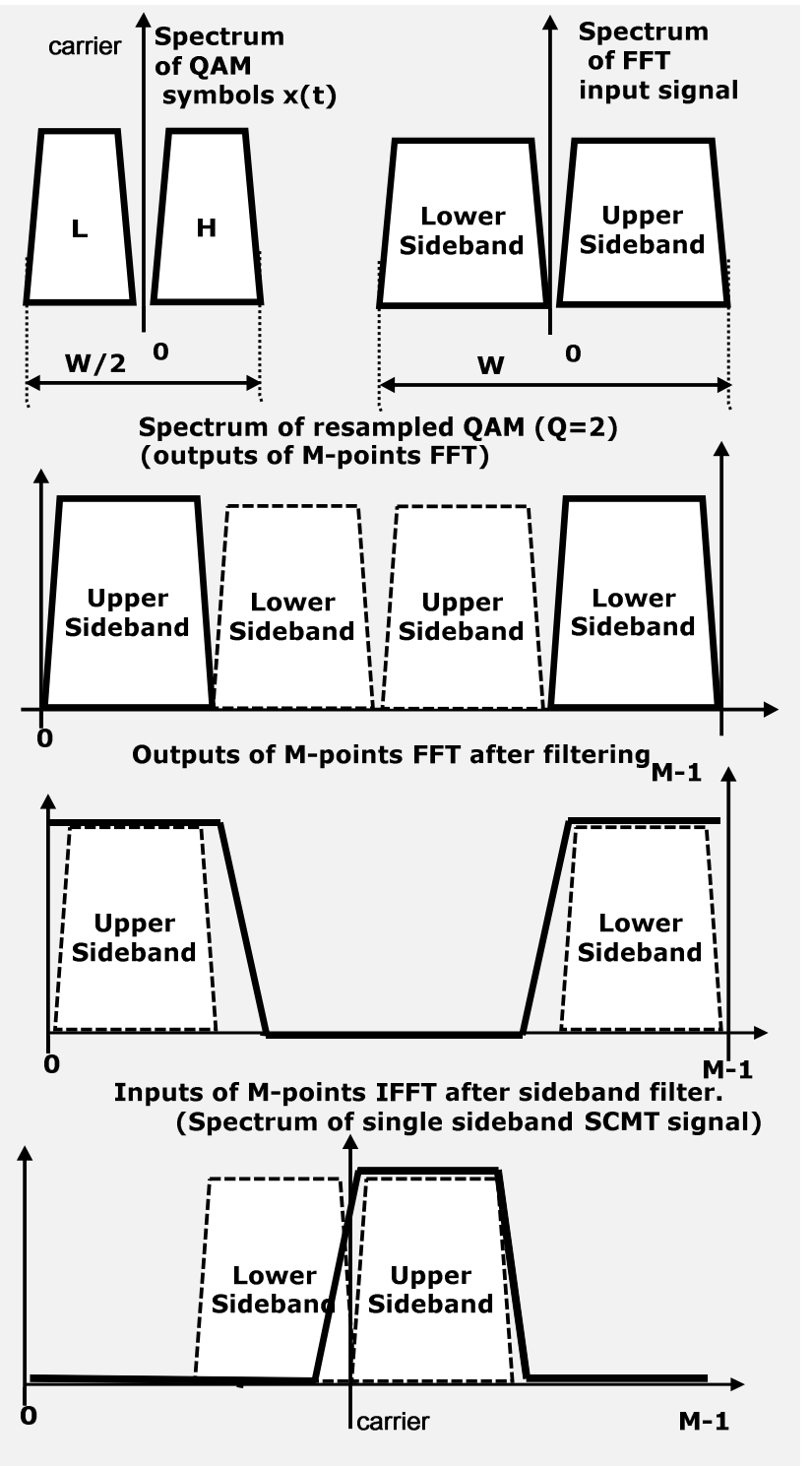

Figure 5: Single sideband SCMT spectrum diagram. 
An important advantage of the proposed SCMT modulation is the possibility of inclusion in the output signal additional impulses, such as the segment sync and frame sync, which are necessary for compatibility with existing ATSC equipment. With the addition of sync pulses, the orthogonality between subcarriers is not broken, as is the case in systems with OFDM. Indeed, in OFDM system the sync pulses may be added only to the output of IFFT by summation subcarriers and said pulses. The spectrum of the sync pulses is uniformly distributed over the entire bandwidth (W) of the transmitted signal that leads to a distortion in amplitude and phase of each subcarrier. In SCMT system the sync pulses can be inserted directly in the input data stream as a specific sequence of bits.

Because this sequence is successively exposed first FFT operation and then the IFFT operation of the same dimension, waveform of signal on FAT input coincides with the envelope of signal on IFFT output. Hence, instead of OFDM, carriers' orthogonality will not fail.

The sync pulses can be easily extracted in the receiver. This process is carried out by correlation of the received signal with the pattern of modulated sync pulse. Figure 6 illustrates the process of segment sync pulse insertion in the ATSC compatible system.

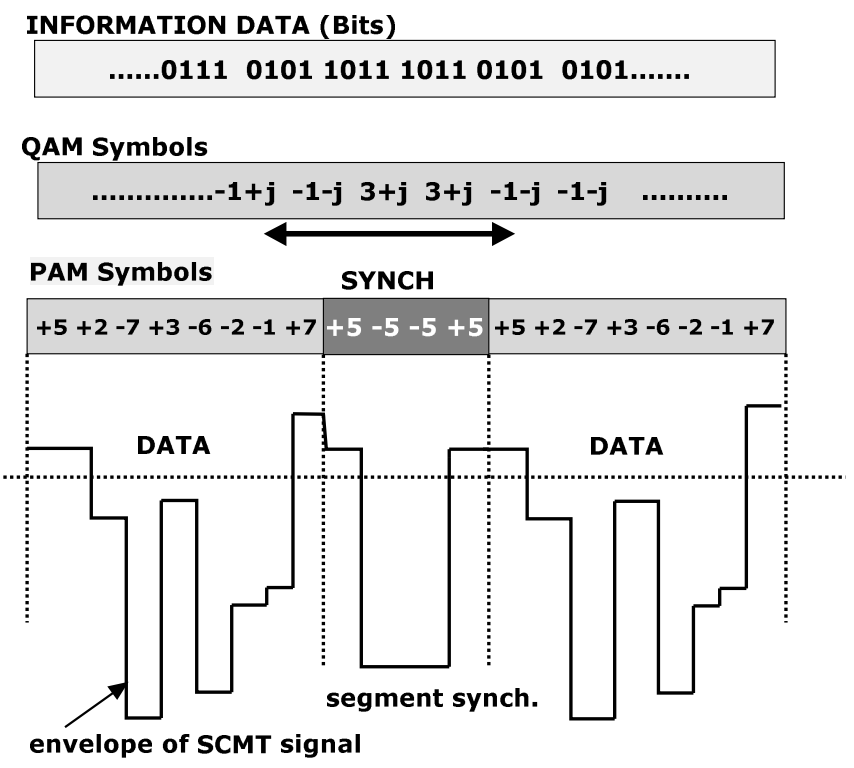

Figure 6: Insertion of segment synchronization pulse.

The described terrestrial TB broadcasting system uses a single-sideband transmission by 2048 carriers in $6 \mathrm{MHz}$ bandwidth. The spectrum of the transmitted signal is similar to the spectrum of the existing system using 8VSB modulation.

The designed scheme uses the same structure of the transmitted signal as the current system. Partially the frame and segment sync signals are identical. Data frames of transmitting signal can include as data fields, which use existing technology in accordance with $\mathrm{A} / 53$ and $\mathrm{A} / 153$ standards, so a data field with SCMT technology.
Figure 7 shows the data field of the transmitted signal of our proposed broadcasting system that will be fully compatible with the existing $\mathrm{A} / 53$ and $\mathrm{A} / 153$ standards. The data field has the standard length $24.2 \mathrm{~ms}$ and comprises a standard field synchronization block FS and exactly 52 data symbols each of them includes one IFFT symbol, cyclic prefix, windowing interval and 6 segment sync impulses. The Data's symbol has a length equal 464us. The cyclic prefix length is about $17 \%$ of data symbol.

The system utilizes the same clock frequency as existing $\mathrm{A} / 53$ systems and can simultaneously transmit standard A/53, A/153 and SCMT data fields.

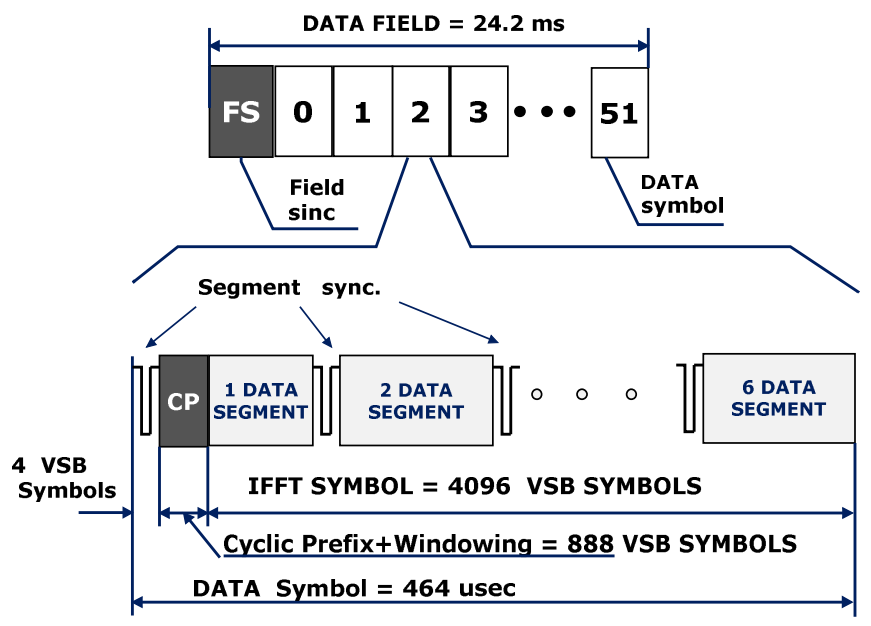

Figure 7: Data field of TV broadcast SCMT system

Instead of standard frames, which use only 8VSB modulation, SCMT frames may carry signals of 16VSB, $32 \mathrm{VSB}$ or $64 \mathrm{VSB}$ modulation. Thus, the SCMT data frames can significantly increase throughput of the TV broadcast system.

\section{MATLAB SIMULATIONS OF THE SCMT SYSTEM}

Below we report results of MATLAB simulations SCMT TV Broadcast system with next parameters:

1. Frequency Bandwidth : $6 \mathrm{MHz}$

2. IFFT/FFT size : $\quad 8 \mathrm{~K}$

3. Number of carriers : $2 \mathrm{~K}$

4. Type of system : single sideband

5. Modulation : $8 \mathrm{VSB}, 16 \mathrm{VSB}, 32 \mathrm{VSB}, 64 \mathrm{VSB}$

Figure 8 shows the spectrum of designing system in the case of the filter with a rectangular shape and bandwidth $\mathrm{W}=$ $2 \mathrm{~K}^{*} \Delta \mathrm{F}$.

Figure 9 shows PAPR of output signal in this case.

Figure 10 shows spectrum of system with raised-cosine filter and roll-off factor 0.25 . 
Figure 11 shows PAPR for output signal a system with raised-cosine filter.

Figure 12 illustrates a low level of the peaks in the output signal of SCMT system

Figure 13 shows a constellation diagram in single sideband SCMT system on the output of QAM decoder for $16 \mathrm{VSB}$ mode of operation

Figure 13 shows a constellation diagram in single sideband SCMT system on the output of QAM decoder for 64VSB mode of operation

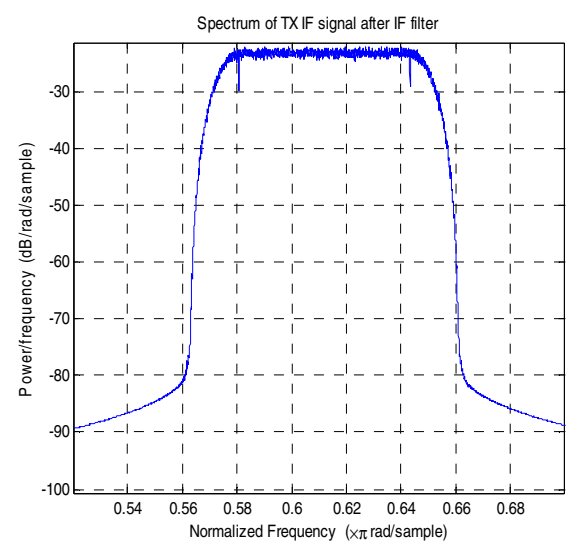

Figure 10: Spectrum of output signal (Raised-cosine filter)

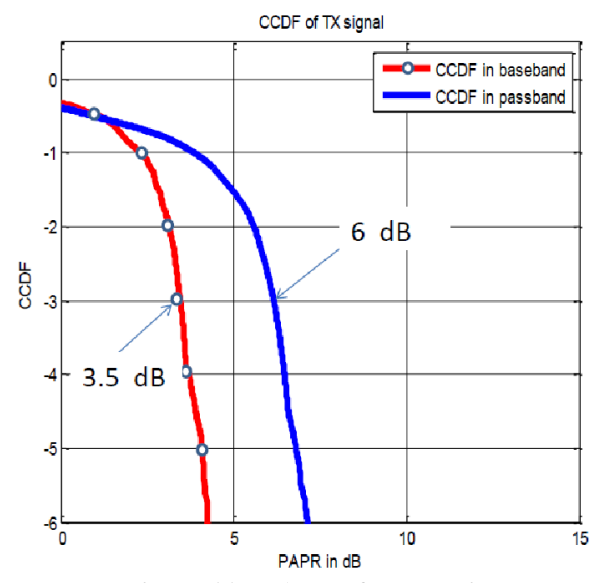

Figure 11: PAPR of output signal (Raised-cosine filter)

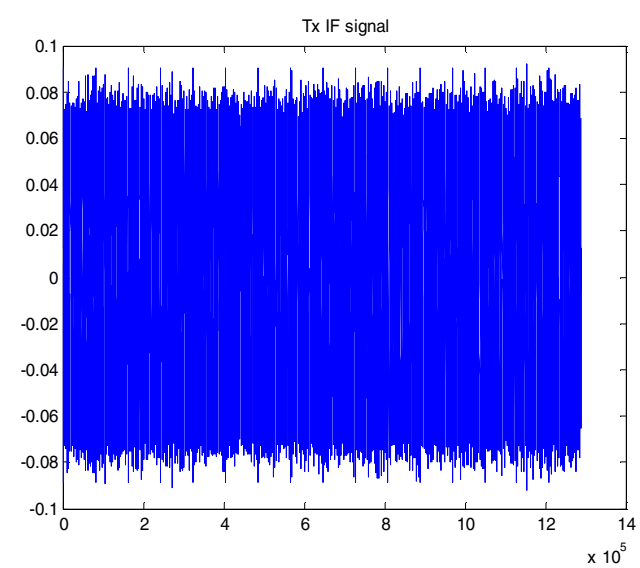

Figure 12: SCMT output signal ( PARP $\sim 6 \mathrm{~dB}$ )

Figure 9: PAPR of output signal (filter with a Rectangular shape) 


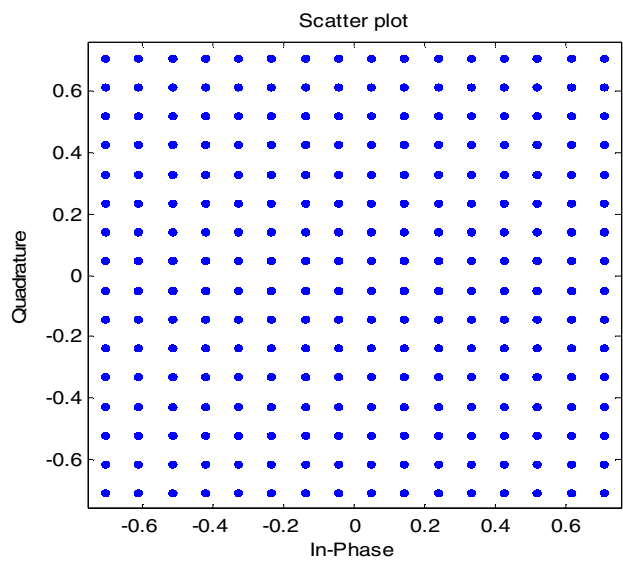

Figure 13: Constellation of received signals. (16VSB mode) Scatter plot

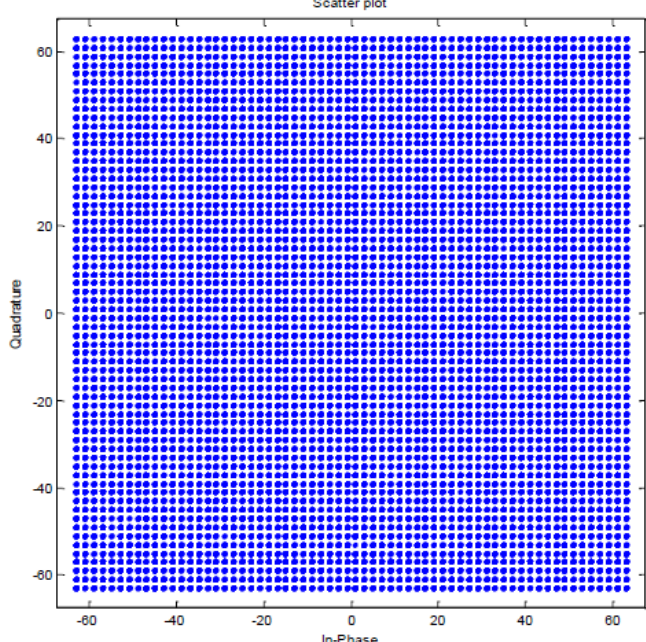

Figure 14: Constellation of received signals. (64VSB mode)

\section{Conclusions}

We have presented the results of a study of a Single Carrier Multi-Tone modulation scheme, which can be successfully used in TV broadcast terrestrial systems and other communication devices that require reliable communication in the presence of reflected signals. Instead of OFDM, proposed system demonstrates low peak-to-average power ratio of the output signal and, therefore, can use low power transmitters. The proposed system provides better performance than known SC-FDMA system and can be used for single sideband transmission. The MATLAB simulations show that SCMT modulation can be a real candidate for a new physical layer for future terrestrial TV broadcasting system and can be used in developing of ATSC 3.0 standard.

\section{REFERENCES}

[1] H. G. Myung, J. Lim, and D. J. Goodman, "Single carrier FDMA for uplink wireless transmission," IEEE Veh. Technol. Mag., vol. 1, no. 3, pp. 30-38, 2006.

[2] N. Benvenuto, R. Dinis, D. Falconer, and S. Tomasin, "Single carrier modulation with nonlinear frequency domain equalization: an idea whose time has come-again," Proc. IEEE, vol. 98, no. 1, pp. 69-96, 2010.

[3] D. Wulich and L. Goldfeld, "Bound of the distribution of instantaneous power in single carrier modulation," IEEE Trans. Wireless Commun., vol. 4, pp. 1773-1778, July 2005.

[4] S. Daumont, B. Rihawi, and Y. Lout, "Root-raised cosine filter influences

on PAPR distribution of single carrier signals," in Proc. 2008 Int. Symp. Commun., Contr. and Signal Process., pp. 841-845.

[5] Hideki Ochiai, "On Instantaneous Power Distributions of Single-Carrier FDMA Signals" IEEE WIRELESS COMMUNICATIONS LETTERS, VOL. 1, NO. 2, APRIL 2012

[6] M. Tanahashi and H. Ochiai, "On the distribution of instantaneous power in single-carrier signals," IEEE Trans. Wireless Commun., vol. 9, pp. 1207-1215, Mar. 2010.

[7] H. Ochiai, "Exact and approximate distributions of instantaneous power for pulse-shaped single-carrier signals," IEEE Trans. Wireless Commun., vol. 10, pp. 682-692, Feb. 2011

[8] T. Frank, A. Klein, and T. Haustein, "A survey on the envelope fluctuations of DFT precoded OFDMA signals," in Proc. IEEE ICC 2008, pp. 3495-3500.

[9] D. Falconer, "Linear precoding of OFDMA signals to minimize their instantaneous power variance," IEEE Trans. Commun., vol. 59, no. 4, pp. 1154-1162, 2011.

[10] D. Falconer, S.L. Ariyavisitakul, A. Benyamin-Seeyar, and B. Eidson, "Frequency Domain Equalization for Single-Carrier Broadband Wireless Systems," IEEE Commun. Mag., vol. 40, no. 4, pp. 58-66, Apr. 2002.

[11]. H. Sari, G. Karam, and I. Jeanclaude, "Transmission Techniques for Digital Terrestrial TV Broadcasting," IEEE Commun. Mag., vol. 33, no. 2, pp. 100-109, Feb.1995.

[12]. J. Lim, H.G. Myung, and D.J. Goodman, "Proportional Fair Scheduling of Uplink Single-Carrier FDMA Systems," to be presented at The 17th Annual IEEE International Symposium on Personal, Indoor and Mobile Radio Communications (PIMRC'06), Helsinki, Finland, Sep. 2006.

[13] 3rd Generation Partnership Project (3GPP); Technical specification group radio access network; Physical layer aspects for evolved UTRA (Release 7)

[14] 3rd Generation Partnership Project; Technical Specification Group Radio Access Network, Physical Layer Aspects for Evolved Universal Terrestrial Radio Access (UTRA), 3GPP TR 25.814 V7.1.0, Sep. 2009.

[15] Evolved Universal Terrestrial Radio Access (E-UTRA); User Equipment (UE) radio transmission and reception (Release 8), 3GPP TS 36.101 v8.7.0, Sep. 2009. 